\title{
||||||||||||||||||||||||||||||||||||||||||||||||||||||||||||||||||.
}

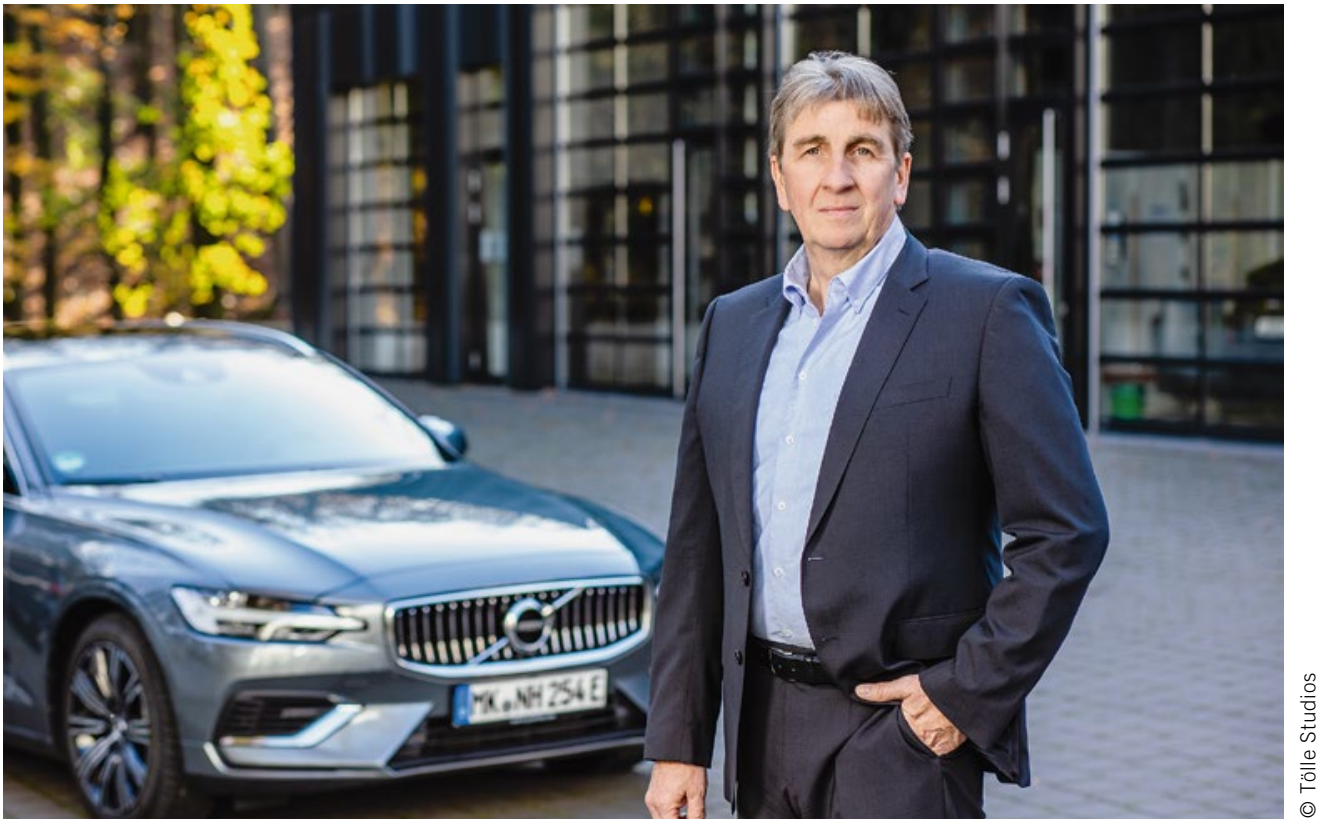

Prof. Dr.-Ing. Wilhelm Hannibal Sprecher des Studiengangs Automotive Engineering der Fachhochschule Südwestfalen

\section{Plug-in-Hybrid wird Mainstream im Pkw-Antrieb}

Auf der MTZ-Fachtagung Experten-Forum-Powertrain 2020 im Oktober in Hanau war man sich in der Diskussion unter Fachleuten weitgehend einig, dass der Hybridantrieb die mittelfristige und langfristige Antriebsstranglösung für die Großserie ist. Nun ist es eher eine Frage des Elektrifizierungsgrads, wie groß die Batterie sein muss und welche Fahrzeugklasse hybridisiert wird. Die Ermittlung des $\mathrm{CO}_{2}$-Footprints über den gesamten Lebenszyklus des Fahrzeugs wird uns zu einer sachlichen Cradle-to-Cradle-Betrachtung führen müssen, wofür die Standards für die Bilanzierung zu definieren sind.

Die Diskussion um den mittelfristigen und langfristigen Pkw-Antrieb muss ergebnisoffen je nach Einsatzfall für die Märkte in der Praxis gesehen werden. Im weniger kostensensiblen Premiumsegment wird sich der Plug-in-Parallelhybrid weiter etablieren. Der Kunde schätzt die damit darstellbare Performance eines Fahrzeugs. Dies unterstreicht auch das derzeitige Angebot im Markt. Die zukünftigen politischen und gesellschaftlichen Entwicklungen werden dieses Segment stark beeinflussen, sodass vermutlich Kontrollmechanismen das rein elektrische Fahren überprüfen.

Im Kleinwagen- und Kompaktwagensegment beträgt die durchschnittliche jährliche Fahrleistung in Deutschland etwa $13.500 \mathrm{~km}$. Hier werden kostengünstige Plug-in-Hybridsysteme zum Einsatz kommen, etwa in einem seriellen Hybrid. Der Verbrennungsmotor wird dann aus Kostengründen entfeinert sein, und da der Kunde mit einer 15 kWh großen Batterie bei einer durchschnittlichen Fahrstrecke unter $60 \mathrm{~km}$ pro Tag bis $\mathrm{zu}$ $80 \mathrm{~km}$ rein elektrisch fahren kann, spielt der verbrennungsmotorische Wirkungsgrad eine untergeordnete Rolle. Mit den langfristig verfügbaren regenerativ erzeugten Kraftstoffen ist dieses Konzept perspektivisch einem BEV überlegen. Denn so fährt man in den Innenstädten emissionsfrei und $\mathrm{CO}_{2}$-neutral über den Brenner zum Gardasee in den Urlaub.

Sicherlich steigt die Vielfalt der parallel existierenden Antriebskonzepte im Pkw, jedoch wird der Plug-in-Hybrid in der Großserie mittelfristig Mainstream werden. Das Umdenken hierzu hat in Fernost schon stattgefunden. Nachdem die Förderung für BEVs in China entfallen ist, rückt der Verbrennungsmotor mit all seinen Anwendungsszenarien wieder in den Fokus der Weiterentwicklung, besonders im Volumensegment. Insbesondere in Korea und China werden enorme Anstrengungen in diesem kostensensiblen Markt unternommen. Eine eigene Patent- und Marktanalyse zeigt auf, dass serielle und leistungsverzweigte Hybridantriebe favorisiert werden.

Die Corona-Situation hat uns gelehrt, dass wir uns hochflexibel den Herausforderungen stellen müssen. Übertragen auf die Fahrzeugantriebstechnik bedeutet das, dass die Kommunikation zwischen Politik, Wirtschaft und Wissenschaft intensiviert werden muss, um unabhängig von Fernost auf dem Weltmarkt wettbewerbsfähig zu bleiben. 